\title{
Agent-based tool to support collaborative KMS in software maintenance process environment
}

\begin{abstract}
Software maintenance (SM) environment is highly complex, knowledge-driven and collaborative. Therefore, knowledge management system (KMS) is critical to provide an environment for sharing and sustaining knowledge among members of SM community of practice $(\mathrm{CoP})$. Several KM retrieval tools are compared to identify the areas that could be improved. One of the important but least available knowledge required by maintainers is the Domain Business knowledge and the relations to the application SM process knowledge. We propose a new tool based on Multi-Agent System (MAS) to address this issue and to assist CoP members to get the right knowledge at the right time.
\end{abstract}

Keyword: Agent technology; KMS; Software maintenance 